\title{
ALTERNATIVAS DE PRETRATAMIENTO TEXTIL: MÉTODO INTEGRADO DE DESCRUDE-BLANQUEO Y BLANQUEO QUÍMICO-ENZIMÁTICO, EVALUACIÓN Y COMPARACIÓN CON EL MÉTODO CLÁSICO
}

\author{
Beatriz Orcón Basilio* ${ }^{* 1}$, Mariel Giraldo Borja ${ }^{2}$, Eduardo Flores Rúa ${ }^{3}$, \\ Anthony Ynca Berrospi ${ }^{4}$
}

\begin{abstract}
RESUMEN
En la Universidad Nacional de Ingeniería (Laboratorio Textil $\left.\mathrm{N}^{\circ} 24\right)$ se realizaron ensayos de pretratamiento para descrude y blanqueo, utilizando como materia prima un tejido de ligamento jersey elaborado con algodón Pima de título 30/1 Ne. Se desarrollaron los procesos de descrude y blanqueo para la obtención de un material hidrófilo con un grado de blancura tal que permita el óptimo teñido de blancos y colores claros. Los ensayos de pre tratamiento se desarrollaron haciendo uso de material de vidrio diverso y agitación manual, mientras que el proceso de tintura se realizó en una máquina Conmatex con movimiento vertical. Para evaluar el grado de blanco, se utilizó el espectrofotómetro Datacolor 550, software Color Tools versión: 1.1.1. Se realizaron tres procesos de blanqueo: clásico o referencial, que consiste en descrude y blanqueo separados; integrado, que considera descrude y blanqueo en un solo baño; y enzimático, donde la enzima activa la formación de peróxido, con fase previa de descrude. Al evaluar absorción y capilaridad, se concluye que el método enzimático tiene resultados similares a los del método clásico, pero mejores que el integrado. La pérdida de peso de los métodos clásico y enzimático es mayor que el método integrado debido a la fase previa de descrude. Los mejores valores para grado de blancura los registró el método clásico seguido del método enzimático y la prueba de teñido comprueba que el color obtenido mediante ambos métodos está en un rango aceptable. El impacto en los efluentes medido a través del $\mathrm{DBO}^{5}$ registró valores menores para el método enzimático.
\end{abstract}

Palabras clave: algodón, descrude, blanqueo, absorción, capilaridad, efluentes.

\footnotetext{
1 Facultad de Ingeniería Química y Textil, Universidad Nacional de Ingeniería, Av. Túpac Amaru 210, Rímac, Lima-Perú, borcon@uni,edu.pe

2 Facultad de Ingeniería Química y Textil, Universidad Nacional de Ingeniería
} 


\title{
TEXTILE PRETREATMENT ALTERNATIVES: INTEGRATED METHOD OF SCOURING-BLEACHING AND CHEMICAL- ENZYMATIC BLEACHING, EVALUATION AND COMPARISON WITH THE CLASSICAL METHOD
}

\begin{abstract}
Pretreatments were made to a Pima cotton jersey knit with yarn number Ne 30/1, with two processes: scouring and bleaching to obtain a hydrophilic material with a whiteness degree that allows dyeing of light colors and whites. The trials were developed at a laboratory level at the National University of Engineering (Textile Laboratory No. 24) using beakers and manual agitation, dyeing was executed on the Conmatex machine with vertical movement and to evaluate the degree of whiteness, the spectrophotometer (Datacolor 550, software Color Tools versión: 1.1.1) was used. Three bleaching processes were carried out: classical (referential) consisting of separate scouring and bleaching stages, integrated that considers scouring and bleaching in a single bath and enzymatic where the enzyme activates the formation of peroxide and has a previous scouring phase. When evaluating absorption and capillarity, it is concluded that the enzymatic method has similar results to those of the classical method and better results than the integrated method. The weight loss of classical and enzymatic methods is greater than the integrated method due to the previous scouring phase. The best values for the degree of whiteness were achieved by the classical method followed by the enzymatic method and the dyeing test proves that the colors obtained from both methods are within an acceptable range. The impact on the effluents measured through $\mathrm{BOD}^{5}$ registered lower values for the enzymatic method.
\end{abstract}

Key words: cotton, scouring, bleaching, absorption, capillarity, effluents.

\section{INTRODUCCIÓN}

El algodón es la fibra natural de mayor importancia mundial como sustrato textil, con él se producen prendas de vestir, artículos para el hogar y productos industriales, su importancia ha decaído con la aparición de las fibras sintéticas, sin embargo, aún representa más del 50 $\%$ de las prendas de vestir. Para muchos tipos de prendas, una prenda de $100 \%$ algodón es señal de calidad garantizada ${ }^{1}$.

La fibra de algodón está compuesta por paredes celulares secas de células vegetales muertas. Luego del desmotado y limpieza mecánica se obtiene aproximadamente $95 \%$ de celulosa. La celulosa en el algodón tiene estructura de polímero lineal de $\beta$-D-glucopiranosa. Los constituyentes no celulósicos generalmente se encuentran en la cutícula, pared primaria y lumen, estos constituyentes son: proteínas $(1,3 \%)$, sustancias pécticas $(1,9 \%)$, cenizas $(1,2 \%)$, ceras $(0,6 \%)$, azúcares $(0,3 \%)$, ácidos orgánicos $(0,8 \%)$, trazas de pigmentos y otros como motas y residuos de semillas y hojas $(1,4 \%)^{2}$. 
El proceso purificador del algodón es el descrude alcalino, que se realiza previo al teñido. Aquí se eliminan componentes no celulósicos del algodón e impurezas agregadas: lubricantes y aceites. La fibra absorbe el álcali, éste neutraliza el grupo carboxilo en la celulosa y en las pectinas. Los grupos hidroxilos en las unidades de glucosa en la celulosa son débilmente ácidos por lo que favorecen el aumento de la concentración del álcali en la fibra y se ataca intensamente a las impurezas. El descrude proporciona hidrofilidad al sustrato por acción del álcali y tensoactivos. Actualmente, se usa el hidróxido de sodio para el descrude que elimina impurezas, pero también degrada la fibra bajo condiciones severas creando fisuras en ellas o disolviendo la cutícula o pared primaria. Estos cambios en el algodón resultan en pérdida de peso (5-10\%) y longitud, alteración en el título del hilo y cambios en la resistencia a la tracción (usualmente un aumento) ${ }^{3}$.

El blanqueo clásico utiliza peróxido de hidrógeno en forma de anión perhidroxi en medio acuoso alcalino ( $\mathrm{pH} 10,5-10,8)$, este anión reacciona con los pigmentos naturales y los oxida ${ }^{4}$. El descrude y blanqueo se realizan en etapas separadas con alto consumo de energía debido a las altas temperaturas necesarias. Para reducir el consumo de energía, se combinan etapas, se reduce el tiempo de reacción o se usan activadores de peróxido a menores temperaturas. La integración de procesos considera productos que potencien la formulación tal que mejoren la remoción y decoloración de impurezas ${ }^{3}$.

FORMACIÓN DE PER HIDROLASA:

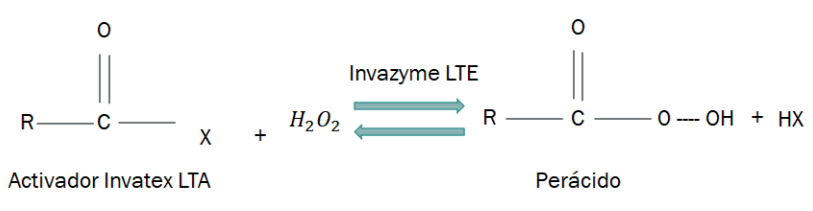

REACCIÓN DE BLANQUEO:

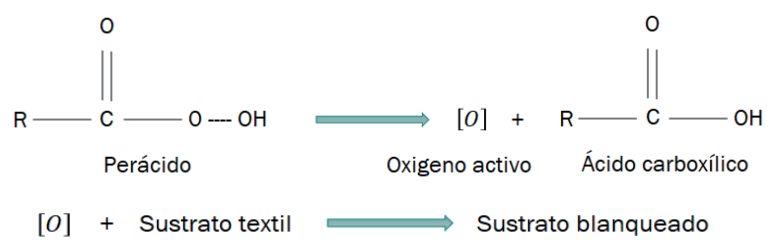

Figura 1. Ecuación del blanqueo enzimático ${ }^{4}$.

El uso de enzimas para el pretratamiento textil tiene ventajas en comparación a los procesos anteriores, se usan como catalizadores a bajas temperaturas, con valores de $\mathrm{pH}$ cercanos al neutro, son biodegradables y el impacto ambiental es $25 \%$ menor que un proceso clásico de blanqueo. En el mecanismo del blanqueo químico enzimático, una enzima del tipo arilesterasa (Invazyme LTE) actúa como catalizador para la formación del perácido, quien libera el oxígeno activo (agente de blanqueo) que reacciona con la fibra para blanquearla. Se muestra la ecuación química con la cual se trabaja este método en la figura $1^{4}$. 
En trabajos anteriores, que analizaron la factibilidad de un tratamiento enzimático para los tres procesos previos: desencolado (aplicable solo a tejidos planos para la remoción de la goma de los hilos de urdimbre), descrude y blanqueo con el uso de una mezcla de enzimas como las amilasas, pectinasas y glucosa oxidasas, el peróxido de hidrógeno se producía enzimáticamente a partir del agente de encolado. Luego, se convertía en la forma de ácido per acético para el blanqueo del tejido de algodón. Sin embargo, el grado de blanco fue medio, pero el grado de absorción fue bueno ${ }^{5}$.

Una de las características más importantes de un efluente es la cantidad de oxígeno necesario para estabilizar, esta cantidad es la demanda bioquímica de oxígeno y es la cantidad de oxígeno requerido para estabilizar el efluente en la presencia de bacterias que consumen los contaminantes químicos ${ }^{6}$.

En la actualidad existen enzimas que pueden reemplazar los procesos de descrude y blanqueo con resultados similares a los procesos clásicos, y que significan una buena alternativa para la reducción de la contaminación? ${ }^{7}$.

\section{PARTE EXPERIMENTAL}

\section{Muestra}

Tejido crudo de ligamento Jersey, con algodón Pima título 30/1 Ne, de gramaje $150 \mathrm{~g} / \mathrm{m}^{2}$, título Ne 30/1.

\section{Condiciones del proceso}

Relación de baño 1:15, que se utilizó debido a que no se contaba con material de vidrio para trabajar relaciones de baño menores. Peso del sustrato: 10 gramos.

Medio de calentamiento y ensayo: Plancha calefactoras con temperatura graduada, vasos de precipitados de vidrio y agitación manual.

\section{Método clásico de descrude y blanqueo}

Se utilizó este método como estándar a motivo de comparación con el método integrado (utilizado actualmente en plantas textiles) y el enzimático (propuesta alternativa ecoamigable). Se inicia con el descrude y a continuación el blanqueo, en ambas etapas se pre calienta la solución a $50{ }^{\circ} \mathrm{C}$ (para disminuir la tensión superficial del agua y facilitar que el textil se sumerja), se agrega el sustrato y se eleva la temperatura a $98{ }^{\circ} \mathrm{C}$ a razón de $3{ }^{\circ} \mathrm{C} / \mathrm{min}$, manteniéndola por 45 min. Para la eliminación del peróxido residual, se utiliza la enzima Catalasa al 0,5 g/l (Globolase $\mathrm{OH}-\mathrm{N})$. 


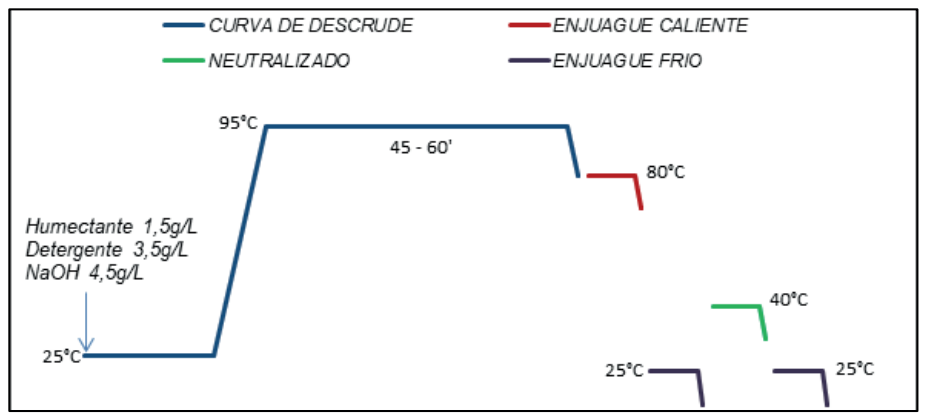

Figura 2. Curva de descrude.

Tabla 1. Receta de descrude.

\begin{tabular}{lll}
\hline PRODUCTO & FUNCIÓN & CANTIDAD \\
\hline Kieralon Wash MFB & Humectante & $1,5 \mathrm{~g} / 1$ \\
Felosan NFGT & Detergente & $3,5 \mathrm{~g} / 1$ \\
Hidróxido de Sodio $50^{\circ} \mathrm{Be}$ & Álcali & $4,0 \mathrm{~g} / 1$ \\
\hline
\end{tabular}

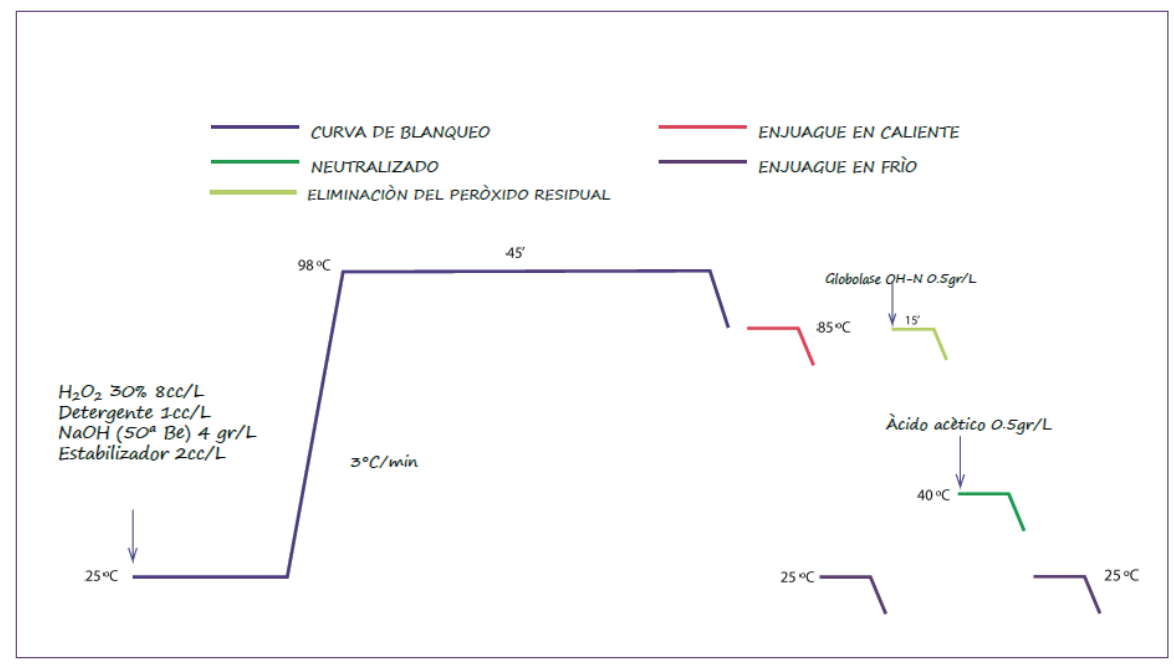

Figura 3. Curva de blanqueo clásico. 
Tabla 2. Receta de blanqueo clásico.

\begin{tabular}{lll}
\hline \multicolumn{1}{c}{ PRODUCTO } & \multicolumn{1}{c}{ FUNCIÓN } & CANTIDAD \\
\hline Peróxido de Hidrógeno al 30\% & Agente de blanqueo & $8 \mathrm{cc} / 1$ \\
Felosan NFGT & Detergente & $1 \mathrm{cc} / 1$ \\
Hidróxido de Sodio $50^{\circ} \mathrm{Be}$ & Álcali & $4 \mathrm{~g} / 1$ \\
Estabilizador SIFA & Estabilizador de peróxido & $2 \mathrm{cc} / 1$ \\
\hline
\end{tabular}

\section{Método integrado}

Se trabaja el descrude y blanqueo en un mismo baño con álcali y peróxido, los \% de ambos sobre el peso del sustrato. Se agrega el álcali a $40^{\circ} \mathrm{C}$ y el textil y se mantiene por 2 minutos, elevándose la temperatura a $60^{\circ} \mathrm{C}$ para ingresar el peróxido. Se eleva la temperatura con gradiente de $3^{\circ} \mathrm{C} / \mathrm{min}$ hasta $98^{\circ} \mathrm{C}$ donde se mantiene por $30 \mathrm{~min}$. Este baño trabaja con humectante, detergente y estabilizador.

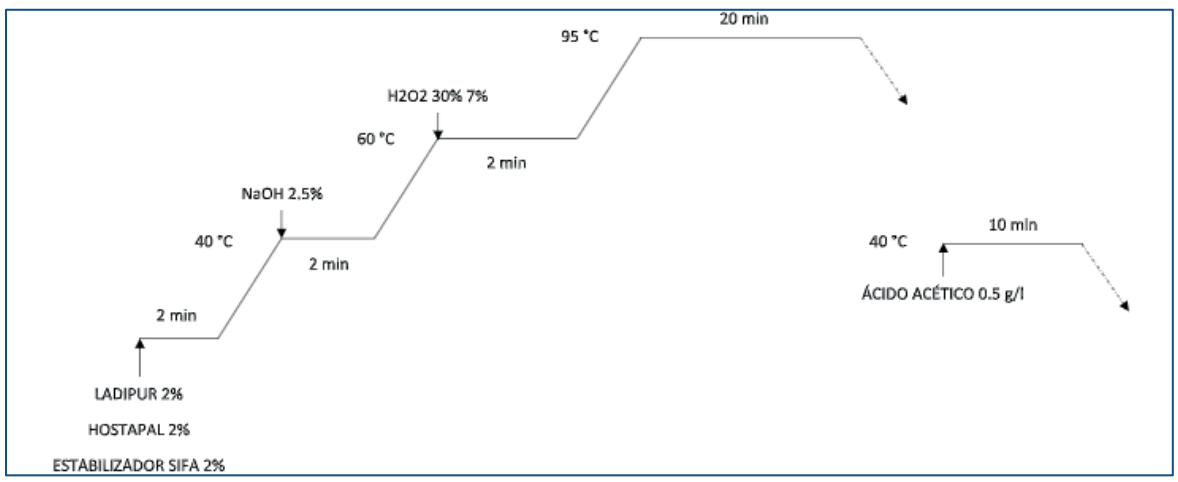

Figura 4. Curva de blanqueo integrado.

Tabla 3. Receta del método integrado.

\begin{tabular}{lll}
\hline \multicolumn{1}{c}{ PRODUCTO } & \multicolumn{1}{c}{ FUNCIÓN } & \multicolumn{1}{c}{ CANTIDAD } \\
\hline Hidróxido de sodio $50^{\circ} \mathrm{Be}$ & Álcali & $4 \%$ \\
Peróxido de hidrógeno $(30 \%)$ & Agente de blanqueo & $7 \%$ \\
Hostapal RMN & Humectante & $1,5 \%$ \\
Felosan NFGT & Detergente & $1,5 \%$ \\
Estabilizador SIFA & Estabilizador de peróxido & $1,5 \%$ \\
\hline
\end{tabular}




\section{Método químico - enzimático}

Consiste en dos etapas, descrude y luego blanqueo enzimático, en baños diferentes. Se trabaja con la enzima de tipo aril-esterasa, que actúa como catalizador para la formación del perácido, que libera oxígeno activo y oxida los pigmentos coloreados de la fibra para blanquearla. El método trabaja con menor concentración de peróxido de hidrógeno y soda cáustica en comparación con el método clásico. Para el blanqueo se agrega detergente, álcali (soda ash), activador de peróxido, peróxido de hidrógeno, y enzima catalizadora. Se precalienta a $40^{\circ} \mathrm{C}$, se agrega el textil y se eleva la temperatura a $65^{\circ} \mathrm{C}$ con gradiente de $2^{\circ} \mathrm{C} / \mathrm{min}$. Luego de 30 minutos, se agrega hidróxido de sodio $1,2 \mathrm{~g} / 1$ y se eleva la temperatura a $98^{\circ} \mathrm{C}$ con gradiente de $1,5^{\circ} \mathrm{C} / \mathrm{min}$., se mantiene por 20 minutos. Luego, en baño nuevo, se agrega catalasa 0,5 $\mathrm{g} / 1$ para eliminar el peróxido residual.

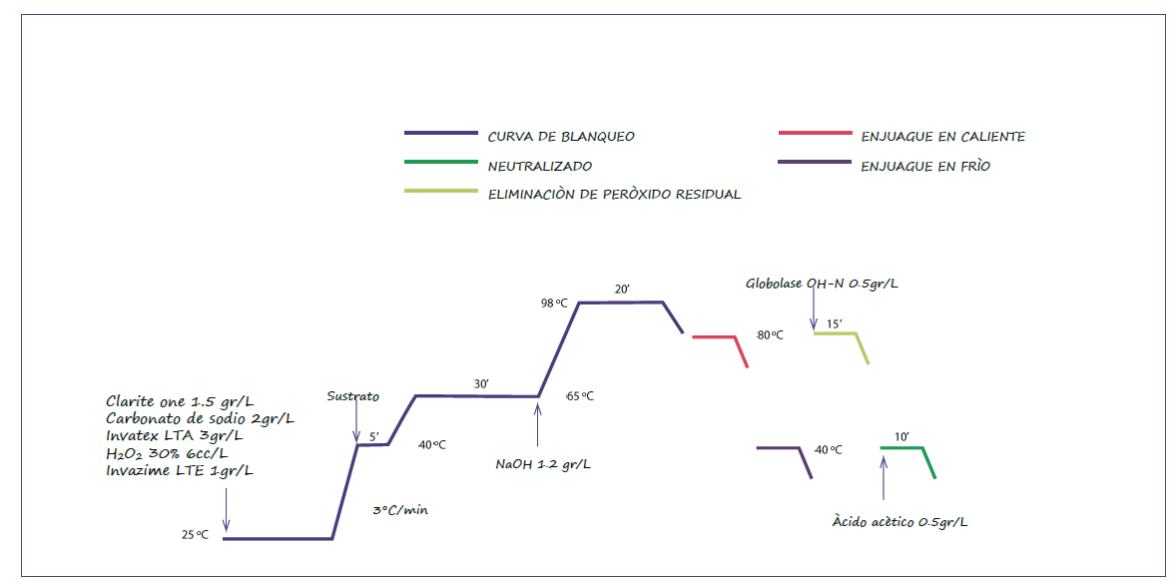

Figura 5. Curva de blanqueo químico-enzimático.

Tabla 4. Receta del método químico-enzimático.

\begin{tabular}{lll}
\hline \multicolumn{1}{c}{ PRODUCTO } & \multicolumn{1}{c}{ FUNCIÓN } & CANTIDAD \\
\hline Clarite One & Detergente & $1,5 \mathrm{~g} / 1$ \\
Carbonato de sodio (soda ash) & Álcali & $2 \mathrm{~g} / 1$ \\
Invatex LTA & Activador de peróxido & $3 \mathrm{~g} / 1$ \\
Peróxido de hidrógeno (30\%) & Agente de blanqueo & $6 \mathrm{cc} / 1$ \\
Invazyme LTE & Enzima catalizadora & $1 \mathrm{~g} / 1$ \\
Hidróxido de sodio $50^{\circ} \mathrm{Be}$ & Álcali & $1,2 \mathrm{~g} / 1$ \\
Globolase OH-N & Enzima catalasa & $0,5 \mathrm{~g} / 1$ \\
\hline
\end{tabular}




\section{Neutralizado}

Posterior a los baños de descrude y blanqueo se realiza un neutralizado con ácido acético glacial a $0,5 \mathrm{~g} / \mathrm{l} \mathrm{a} 40^{\circ} \mathrm{C}$, seguido por enjuagues en caliente y frío.

\section{Eliminación del peróxido residual}

Posterior a los baños que usan peróxido de hidrógeno, se realiza la conversión hacia agua y oxígeno mediante el uso de $0,5 \mathrm{~g} / \mathrm{l}$ de catalasa y enjuagues en tibio y frío.

\section{Prueba de teñido}

Las muestras pre tratadas y blanqueadas son teñidas con colorante reactivo Azul Everzol SAM con una relación de baño $1: 15$. La temperatura inicia a $40^{\circ} \mathrm{C}$, en presencia de auxiliares (secuestrante, Sirrix 1g/l; humectante, Hostapal HRM 1g/l). Después de 10 min se adiciona el colorante, $1 \%$ sobre el peso del sustrato. Luego del mismo tiempo, para fijar el colorante, se adiciona una décima parte de carbonato, elevando la temperatura a razón de $1,5{ }^{\circ} \mathrm{C} / \mathrm{min}$ hasta a los $60^{\circ} \mathrm{C}$. Luego de intervalos de veinte minutos, se adiciona el resto de carbonato en partes de tres y seis décimos, para permanecer en agitación durante 45-60 minutos. El proceso concluye con el respectivo neutralizado y enjuagues varios.

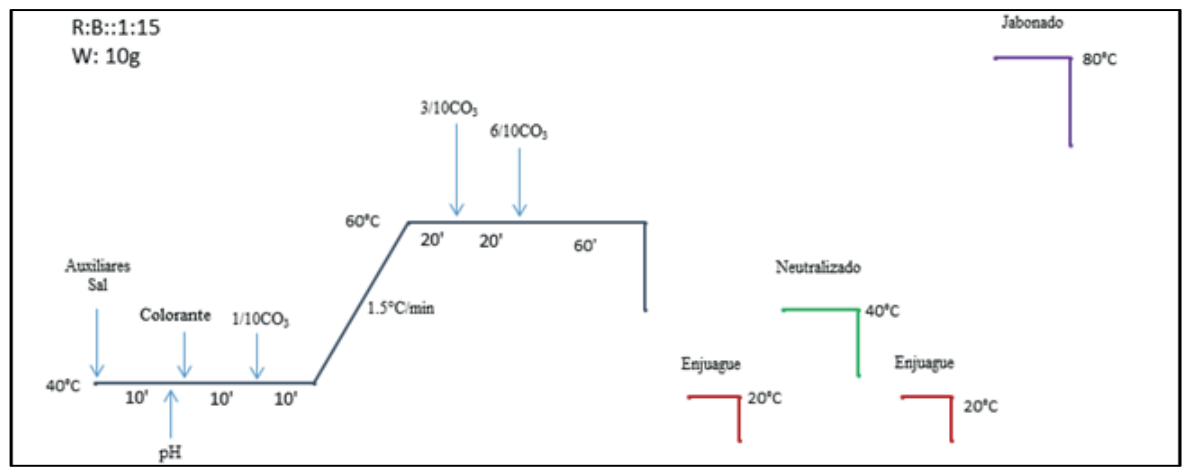

Figura 6. Curva de teñido con colorantes reactivos.

\section{RESULTADOS Y DISCUSIÓN}

\section{Demanda bioquímica de oxígeno (DBO5)}

Es la cantidad de oxígeno en $\mathrm{mg} / \mathrm{l}$ necesaria para descomponer la materia orgánica presente mediante acción de los microorganismos aerobios presentes en el agua.

Tabla 5. Tabla comparativa de $\mathrm{DBO}_{5}$.

\begin{tabular}{lc}
\hline MÉTODO & DBO $_{\mathbf{5}} \mathbf{( m g / \mathbf { l } )}$ \\
\hline Integrado & 1179 \\
Clásico & 717 \\
Enzimático & 519 \\
\hline
\end{tabular}


La DBO del baño residual del blanqueo enzimático es la menor de los tres métodos, lo que indica que el consumo del oxígeno disuelto por la materia orgánica presente en el baño es el menor ya que existe menor presencia de materia orgánica en el baño residual.

La DBO del baño residual del método integrado es la mayor de los tres debido a que hay una combinación de dos procesos: descrude y blanqueo en un mismo baño, altamente alcalino que provocan mayor presencia orgánica descompuesta en el baño.

\section{Grado de blancura}

Haciendo uso del espectrofotómetro DATACOLOR, que mide los valores triestímulos, obtenemos el valor de blancura medido en grados Berger.

La tabla 6 muestra el resultado del grado de blanco para los tres métodos de pretratamiento.

Tabla 6. Tabla comparativa del grado de blancura en ${ }^{\circ} \mathrm{Be}$.

\begin{tabular}{cc}
\hline MÉTODO & GRADO BERGER \\
\hline Clásico & 78,18 \\
Enzimático & 77,54 \\
Integrado & 71,28 \\
\hline
\end{tabular}

Se observa en la figura 7 que la curva longitud de onda vs reflectancia es muy similar para los descrudes clásico y enzimático, dando un grado de blanco aceptable.

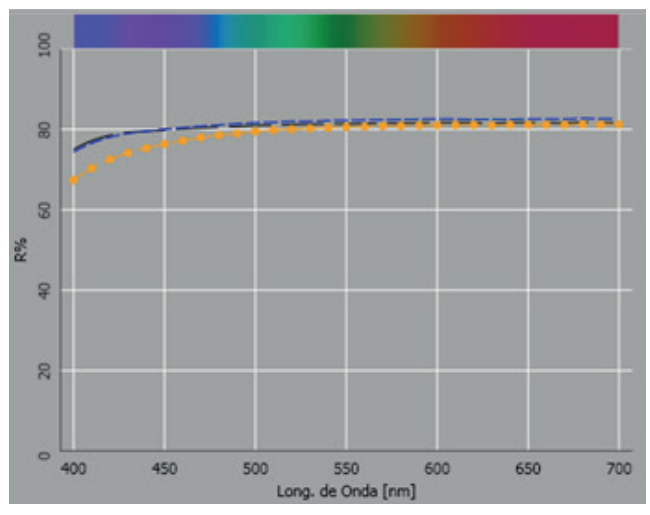

Figura 7. Longitud de onda vs reflectancia: ----- Clásico, ---- Integrado, ---- Enzimático 
Tabla 7. Descripción del blanco en la escala CIELab.

\begin{tabular}{|c|c|c|c|}
\hline ESTÁNDAR & LOTE & LECTURA CIE Lab & DESCRIPCIÓN \\
\hline Clásico & Enzimático & Pasa & $\begin{array}{l}\text { Más claro, más verde y más } \\
\text { amarillo }\end{array}$ \\
\hline Clásico & Integrado & Falla & $\begin{array}{l}\text { Más oscuro, más verde y más } \\
\text { amarillo }\end{array}$ \\
\hline
\end{tabular}

\section{Medida del pH}

La medición del pH se realizó en la etapa del blanqueo para los tres métodos después de terminado el proceso, es decir, en la etapa final considerando las dosificaciones de carbonato y soda posteriormente, en los casos que aplica.

Tabla 8. Tabla comparativa de $\mathrm{pH}$.

\begin{tabular}{cc}
\hline MÉTODO & $\mathbf{p H}$ \\
\hline Integrado & 11 \\
Clásico & 12 \\
Enzimático & 9 \\
\hline
\end{tabular}

\section{Evaluación del grado de descrude}

\section{Determinación de la pérdida de peso}

Se realizó la evaluación a los tres procedimientos de blanqueo, aplicando lo siguiente:

$$
\% \text { Peso perdido }=\frac{\text { Peso }_{\text {antes del descrude }}-\text { Peso }_{\text {después del descrude }}}{\text { Peso }}(100 \%)
$$

Tabla 9. Tabla comparativa del porcentaje en pérdida de peso total incluyendo descrude.

\begin{tabular}{lcccc}
\hline MÉTODO & $\begin{array}{c}\text { PESO } \\
\text { INICIAL }\end{array}$ & $\begin{array}{c}\text { PESO } \\
\text { FINAL }\end{array}$ & $\begin{array}{c}\text { \% PÉRDIDA DE } \\
\text { PESO BLANQUEO }\end{array}$ & $\begin{array}{c}\text { \% PÉRDIDA } \\
\text { DE PESO TOTAL }\end{array}$ \\
\hline Enzimático & 10,00 & 9,808 & 1,25 & 4,13 \\
Integrado & 10,00 & 9,653 & 2,78 & 2,78 \\
Clásico & 10,00 & 9,761 & 1.75 & 4,63 \\
\hline
\end{tabular}


Prueba de absorción (Hidrofilidad: Norma AATCC TM 79 - 2014)

Este método está diseñado para medir la absorción de soluciones en los sustratos textiles, mediante el tiempo que tarda una gota de agua con colorante en la superficie del tejido, para ser absorbida completamente. Según esta norma, la muestra se coloca en la parte superior de un vaso de precipitado, dejando caer una gota a $1 \mathrm{~cm}$ de la superficie del tejido. Se adaptó el método colocando la tela sin tensión sobre un vaso de precipitados fijado con una liga para evitar las arrugas. Se midió el tiempo que la gota se absorbe en el tejido.

Tabla 10. Comparación del tiempo de absorción de una gota de agua.

\begin{tabular}{cc}
\hline MÉTODO & TIEMPO DE ABSORCIÓN (SEG.) \\
\hline Integrado & 2 \\
Enzimático & 1 \\
Clásico & 1 \\
\hline
\end{tabular}

Prueba de capilaridad (Capilaridad: NORMA AATCC 197-2013)

Este método se usa para evaluar la facilidad con la que una solución se transporta a lo largo del sustrato textil. Se coloca la tela de forma vertical, en sentido de las columnas o cursas (tejido de punto) y se permite el ascenso del agua con colorante, midiendo la altura que alcanza sobre la tela a los 30 minutos. Se trabajó con una tela de $5 \mathrm{~cm}$ de ancho y $10 \mathrm{~cm}$ de alto y se sumergió en una solución de colorante reactivo Turqueza Everzol ED.

Tabla 11. Comparación del ascenso de agua con colorante en los tres métodos.

\begin{tabular}{cc}
\hline MÉTODO & ASCENSO DE COLOR (cm) \\
\hline Enzimático & 8,90 \\
Clásico & 8,50 \\
Integrado & 7,30 \\
\hline
\end{tabular}

\section{Evaluación de tintura}

Se trabajó la misma receta de teñido a las mismas condiciones en los tres métodos de blanqueo: Clásico-Integrado-Enzimático.

Se realizaron pruebas en el espectrofotómetro DATACOLOR, para poder revisar la reproducibilidad de color, tomando como estándar el teñido del método clásico. 
Tabla 12. Descripción de los resultados en función al valor CIE Lab.

\begin{tabular}{cccc}
\hline ESTÁNDAR & LOTE & $\begin{array}{c}\text { Lectura } \\
\text { CieLab }\end{array}$ & DESCRIPCIÓN LOTE \\
\hline Clásico & Enzimático & Pasa & Más oscuro, más verde y más azul \\
Clásico & Integrado & Falla & Más oscuro, más verde y más amarillo \\
\hline
\end{tabular}

Tabla 13. Tabla de diferencia de color total CIE Lab.

\begin{tabular}{lllcrrc}
\hline LOTE & CIE Da & CIE Db & CIE DC & CIE DH & CIE DE & LECTURA \\
\hline Enzimático & $-0,06$ & $-0,43$ & 0,43 & 0,07 & 0,22 & Pasa \\
Integrado & $-0,03$ & 0,35 & $-0,33$ & $-0,13$ & 0,37 & Pasa \\
\hline
\end{tabular}

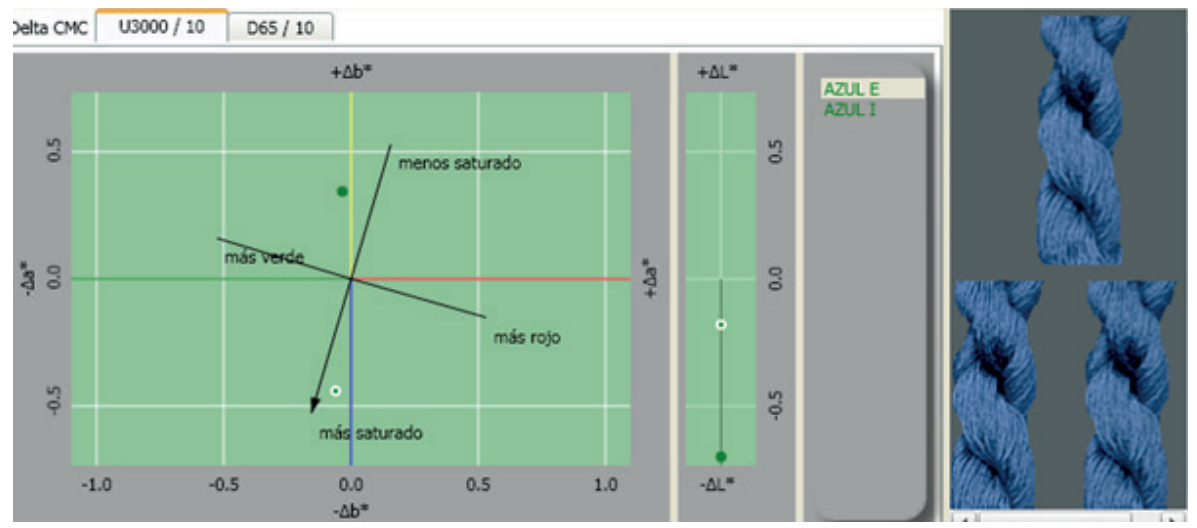

Figura 8. Espacio de color CIE LAB. 


\section{CONCLUSIONES}

El método clásico obtuvo un grado de blanco de $78,18^{\circ} \mathrm{Be}$, el cual es casi un punto superior al del método de descrude/blanqueo enzimático. Por otro lado, el método integrado resulta con un grado de blanco de $71,28^{\circ} \mathrm{Be}$. Adicionalmente se evaluaron las muestras con la escala CIELab, tomando como estándar el blanco obtenido con el método clásico. Se concluye que el método descrude/blanqueo enzimático es aceptable como alternativa para colores claros y blancos ópticos y por el contrario el blanco obtenido con el método integrado solo para tonos medios.

Con los resultados de $\mathrm{DBO}_{5}$ se concluye que el proceso de pretratamiento con el método enzimático tiene un impacto ambiental menor al método clásico, ya que es $27 \%$ menor que éste, y más del $100 \%$ menor que el del método integrado.

Una preocupación de las empresas textiles, es el alto \% de mermas, que implica costos por pérdidas de material. El método descrude/blanqueo enzimático resulta con una pérdida de peso total de $4,13 \%$ y solo en el proceso de blanqueo, su pérdida de peso es de $1,25 \%$. El menor valor para pérdida de peso total, lo registra el blanqueo integrado. El método clásico referencial, solo para el grado de blancura, registra una pérdida de peso del 4,63\% total y $1,75 \%$ por el blanqueo. Se concluye que el blanqueo enzimático favorece la conservación de la masa celulósica en la fase de blanqueo.

Los mejores valores de grado de absorción y capilaridad fueron los correspondientes a los métodos descrude/blanqueo enzimático y al clásico, lo que significa que el grado de limpieza de las paredes celulares y accesibilidad de los capilares es similar, lo que se traduce en una buena hidrofilidad del textil.

Con los resultados de la evaluación tintórea se concluye que el método de descrude/ blanqueo enzimático obtienen resultados muy similares al método clásico, por lo tanto lo podría reemplazar.

Como conclusión final el método propuesto de descrude/blanqueo enzimático resulta en menor impacto ambiental, grado de blanco muy similar y resultados de tintura aceptables por lo que una siguiente propuesta de trabajo sería la de eliminar el descrude y reemplazarlo con un bio tratamiento enzimático integral.

\section{AGRADECIMIENTOS}

A la Facultad de Ingeniería Química y Textil de la Universidad Nacional de Ingeniería, por el uso de sus instalaciones y la formación académica recibida. Finalmente, un agradecimiento a los ingenieros Ángela Ortiz e Iván Arana por su apoyo incondicional. 


\section{REFERENCIAS BIBLIOGRÁFICAS}

1. Cassidy T, Goswami P. editors. Textile and Clothing Design Technology. Boca Raton, Florida: CRC Press; 2017.

2. Menachem L. editor. Handbook of Fiber Chemistry. Boca Raton, Florida: CRC Press; 2006.

3. Karmakar S. Chemical Technology in the Pre-treatment Processes of Textiles. Amsterdam: Elsevier Science B.V; 1999.

4. Siddiquee AB, Bashar M, Sarker P, Tohfa T, Hossan A, Ibrahim Azad I, et al. Comparative study of conventional and enzymatic pretreatment (scouring \& bleaching) of cotton knitted fabric. Int J Eng Technol. 2014; 3(1):37-43

5. Spicka N, Forte P. Complete enximatic pre-treatment of cotton fabric with incorporates bleach activator. Text Res J. 2013; 83(6):566-573

6. Fan Q. Chemical Testing of Textiles. Boca Raton, Florida: CRC Press; 2005.

7. Fakin D, Golob D, Stjepanovič Z. The Effect of Pretreatment on the Environment and Dyeing Properties of a Selected Cotton Knitted Fabric. Fibres Text East Eur. 2008; 16(2):101-104. 